CORRIGENDUM

\title{
A calcium-activated nucleotidase secreted from Ostertagia ostertagi 4 th-stage larvae is a member of the novel salivary apyrases present in blood-feeding arthropods - CORRIGENDUM
}

D. S. ZARLENGA, A. J. NisBeT, L. C. GASBARRE and W. M. GARRETT

doi:10.1017/S0031182010001241, Published by Cambridge University Press, 1 September 2010.

The order of sequences that appear in Figure 1B should read as follows:

Oos-APY -1

$\mathrm{Ll}$

$\mathrm{Hu}$

$\mathrm{Xl}$

$\mathrm{Ce}$

$\mathrm{Cl}$

$\mathrm{Dm}$

$\mathrm{Mm}$

$\mathrm{Pp}$

$\mathrm{Rn}$

The correct version of Figure 1B is presented overleaf. 


Oos-APY-1
$\mathrm{Ll}$
$\mathrm{Hu}$
$\mathrm{Xl}$
$\mathrm{Ce}$
$\mathrm{Cl}$
$\mathrm{Dm}$
$\mathrm{Mm}$
$\mathrm{Pp}$
$\mathrm{Rn}$

Oos-APY-1

L1

$\mathrm{Hu}$

$\mathrm{Xl}$

$\mathrm{Ce}$

Dm

Mm

$\mathrm{P}_{\mathrm{p}}$

Rn

Oos-APY-1
$\mathrm{Ll}$
$\mathrm{Hu}$
$\mathrm{Xl}$
$\mathrm{Ce}$
$\mathrm{Cl}$
$\mathrm{Dm}$
$\mathrm{Mm}$
$\mathrm{Pp}$
$\mathrm{Rn}$

Oos-APY-1
$\mathrm{Ll}$
$\mathrm{Hu}$
$\mathrm{Xl}$
$\mathrm{Ce}$
$\mathrm{Cl}$
$\mathrm{Dm}$
$\mathrm{Mm}$
$\mathrm{Pp}$
$\mathrm{Rn}$

Oos-APY-1

Ll

$\mathrm{Hu}$

$\mathrm{X}$ I

$\mathrm{Ce}$

Dm

$\mathrm{Mm}$

$\mathrm{Pp}_{\mathrm{p}}$

Rn

Oos-APY-1

L1

$\mathrm{Hu}$

$\mathrm{Xl}$

Cl

Dm

Mm

$\mathrm{Pp}$
$\mathrm{Rn}$

Oos-APY-1
$\mathrm{Ll}$
$\mathrm{Hu}$
$\mathrm{Xl}$
$\mathrm{Ce}$
$\mathrm{Cl}$
$\mathrm{Dm}$
$\mathrm{Mm}$
$\mathrm{Pp}$
$\mathrm{Rn}$

50

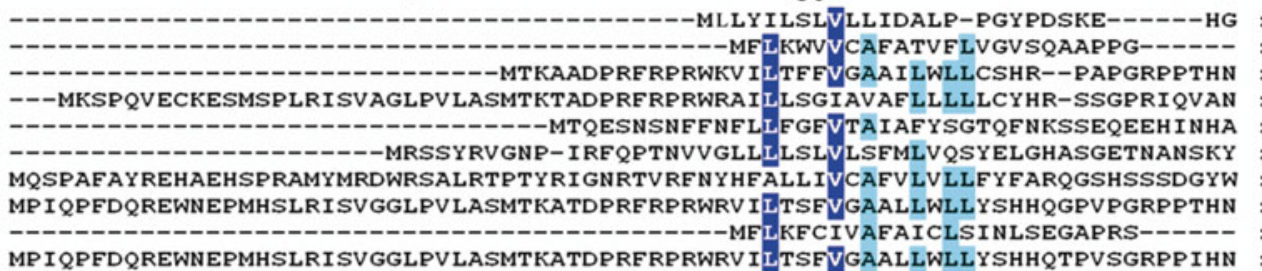

100

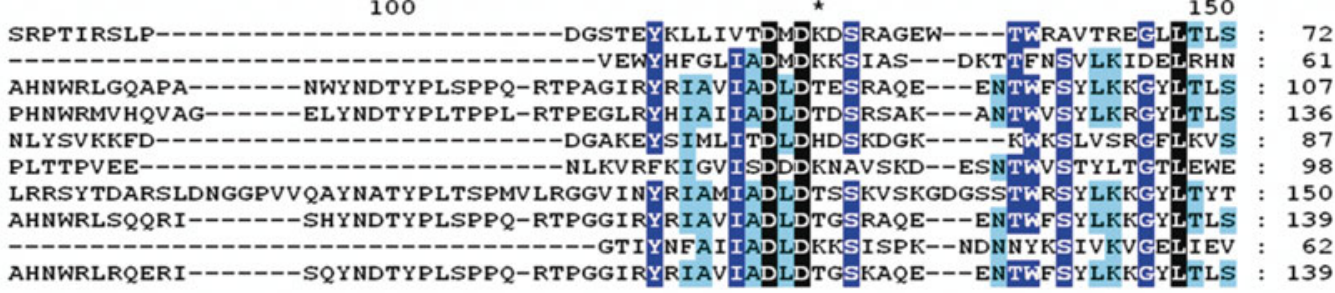
$+$ 200 PDMTHVSI TWDEKSERNLTSSMN I KGRAMELSDLSVFHNRI LTPDDRTGLISEIKNN-KMIPWVEZNSEPENTTS TKTDQY I YVRSR-VKKPVSTRYGFKGREAELSEIVVENNKLYTVDDKSEITFRITKDGKLFPWVILADADGQRPD : 135 DSGDKVAVEWDK-DHGVLESHLAEKGRGMELSDLIVENGKL Y SVDDRTGVVYQIEGS-KAVPWVILSDGDGTVEK : 180 SSGDHVAVEWEK-EDIVLETHLAEKGRGMELSEL IVFNGKL Y SVDDRTGVVYRIEGS-KAVPWVILTDGDGTVGK : 209 ADHKHAD I HFDKD SEYYVDTN I AAGGRAMELSDLAVENGKL Y I IDDRT EL I Y ISDK-KALPWVLLNDEPGNVVK : 161 KSTDKITVQWDKGNEKKVKSKYSYGGRGMELSELVTFNGNLLTFDDRTGLVYILKDD-KVYPWVVLADGDGKNSK: 172 VARSEIQISWDDGAP IVLESAF ALKGRGMELSELVTFNGRLLTFDDRT GL I YEIVND-KP I PWV ILLDEDGHSAK : 224 DSGDRV SVEWDK-DHGVLESHLAEKGRGMELSDLIVENGKL Y SVDDRT GV I YQIEGT-KAVPWVILSDGDGTVEK : 212 G--DKY

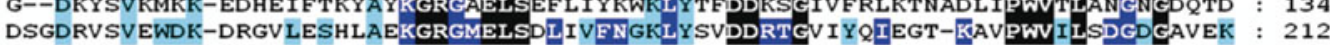

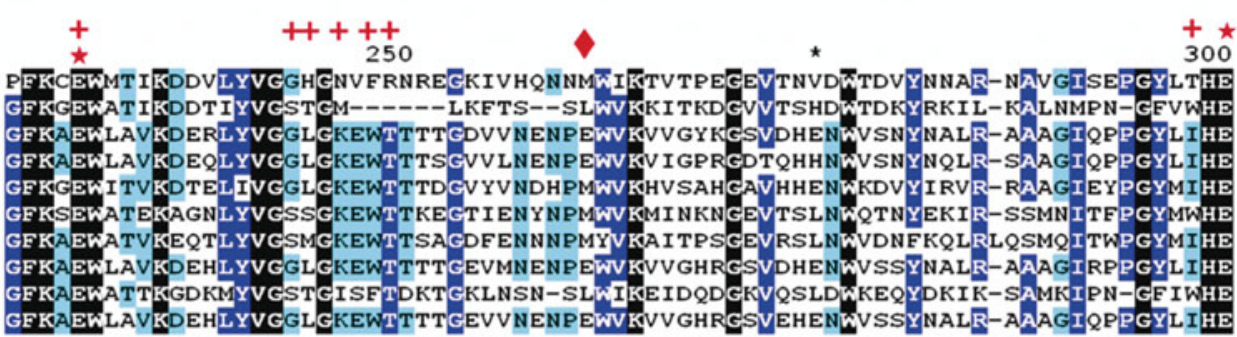

300

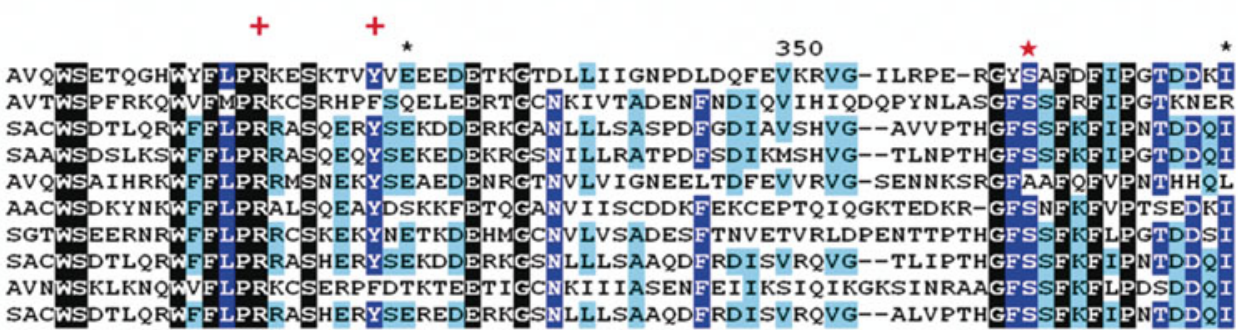

350

$\star$

293

\section{0} $+$

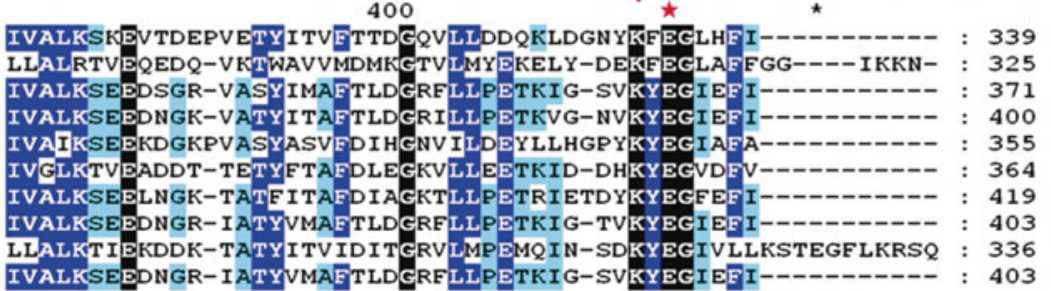

Zarlenga, D. S., Nisbet, A. J., Gasbarre, L. C. and Garrett, W. M. (2011). A calcium-activated nucleotidase secreted from Ostertagia ostertagi 4th-stage larvae is a member of the novel salivary apyrases present in blood-feeding arthropods. Parasitology, 138, 333-343. doi: 10.1017/S0031182010001241. 